\title{
ELECTRON INJECTION IN SEMICONDUCTOR DRIFT DETECTORS
}

\author{
P. Reliak \\ Brooihaven National Laboratory, Upton NY 11973
}

BNL- -45373

DE91 004796

E. Gatti, A. Longoni, M. Sampietro, A. Castoldi

CEQSE CNR, Politecnico di Milano, Piazza Leonardo da Vinci 92, Milano*

\section{A. Vacchi}

Rockefeller University, New York, NY 10021

\begin{abstract}
The paper reports the first successful results of a simple MOS structure to inject electrons at a given posilion in Silicon Drift Detectors. The structure allows on -line calibration of the drift velocity of electrons within the detector. The calibration is a practical method to trace the temperature dependence of the electron mobility.

Severnl of these injection structures can be implemented in silicon drift detectors without additional steps in the rabrication process.
\end{abstract}

\section{INTRODUCTION}

Silicon drift detectors are a relatively new kind of semiconductor detector able to provide very precise position and ionization measurements with a very modest amount of electronics. A perspective view of the drift detector [1] is shown in Fig. 1. In principle, the electric field of thedrift detector forces electrons liberated by an ionizing particle to drift parallel to the large semiconductor surface to the anode. The transit time of electrons inside the detector measures the distance of an incident particle from the anode.

As a direct consequence of this electron transport method, the anode capacitance is much lower than that of a classical semiconductor detector of the same dimensions. The amplifier noise can be made much smaller, which is the main reason behind the excellent position resolution obtained from silicon drift detectors.

The silicon drift detectors are quite sensitive to temperature changes. The drift velocity in silicon depends on the absolute temperature as $T^{-2.4}$ [2]. Due to the precisionof the drift coordinate measurement, silicon drift

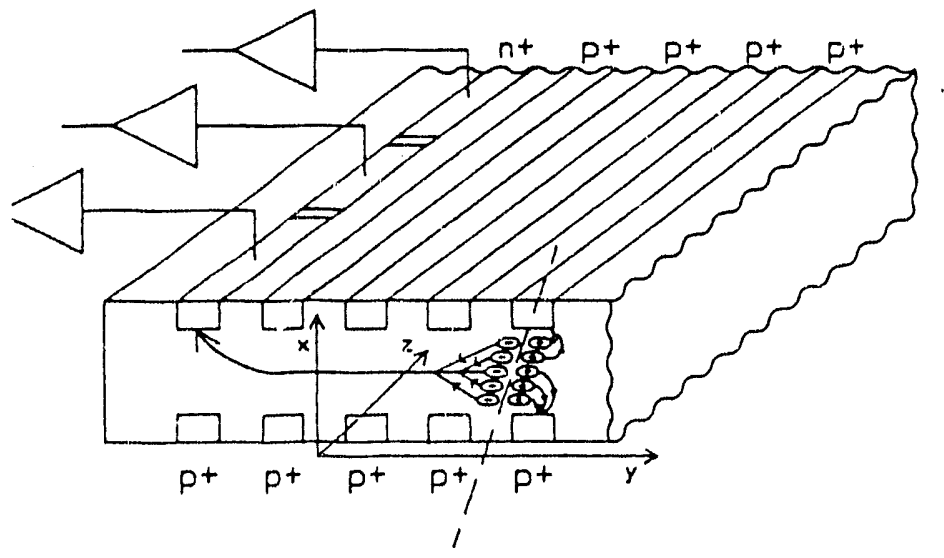

Figure 1: Perspective view (not to scale) of a semiconductor drift detector. Electrons created by an ionizing particle are transported long distances parallel to the detector surface. The anode is divided into short segments to measure the coordinate perpendicular to the drift direction.

detectors are sensitive to temperature variations of about $0.1 \mathrm{~K}$. Stabilizing the overall temperature to better than $0.1 \mathrm{~K}$ may not be practical. Rather, it seems easier to calibrate the drift velocity.

\section{ELECTRON INJECTOR}

\section{A. Experimental Results}

A practical calibration of the drift velocity must be accomplished by simple and reproducible means, ideally by pulsing an electrode at a given distance from the anode. Such an injecting electrode was implemented on a silicon drift detector proposed for use in the UA6 experiment at the CERN SPPS. [3] The injector is a small metallic gate deposited on the silicon dioxide present 


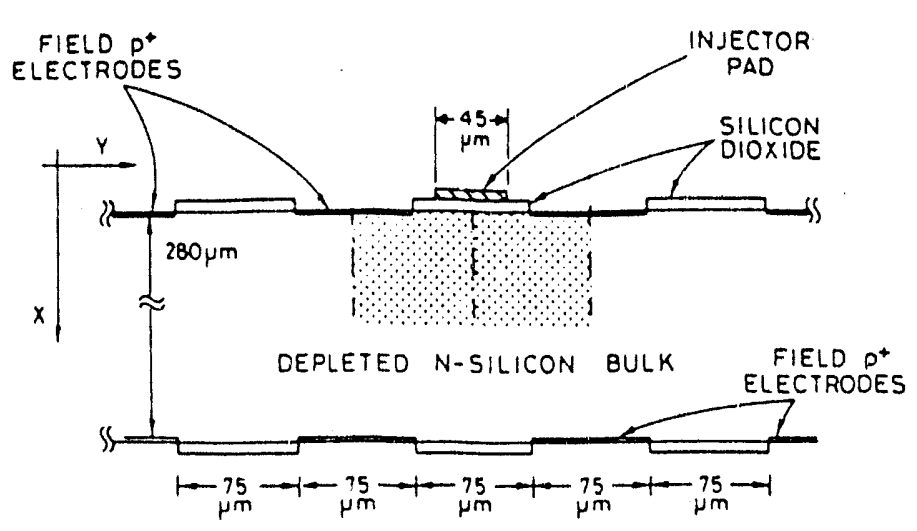

Figure 2: Cross section of the Silicon drift detector in the injector region. The $z$-direction is perpendicular to the plane.

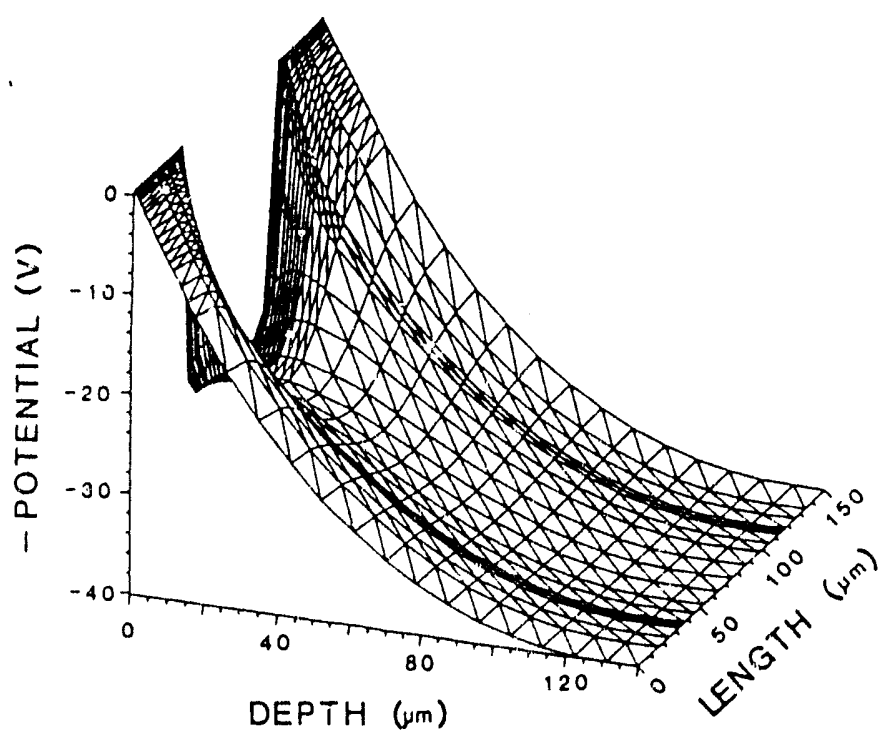

Figure 3: Potential energy for electrons calculated within the dotted area on the previous Figure.

between the field $p^{+}$strips, at the desired position. A cross section of the detector around an injector pad is shown in Fig. 2. The injector is a metal on silicon (MOS) capacitor. The injection mechanism is related to the actual shape of the potential energy for electrons in a silicon drift detector with working biases. The shape of the potenvial energy for electrons is shown in Fig. 3 for the ciotted region of Fig. 2.

The positive charge at the $\mathrm{Si}-\mathrm{SiO}_{2}$ interface causes a downward bending of the potential at the interface. There are many electrons confined in the surface region extending up to the saddle point. [4] The number of accumulated electrons in this surface region is the result of a dynamic equilibrium between a surface and

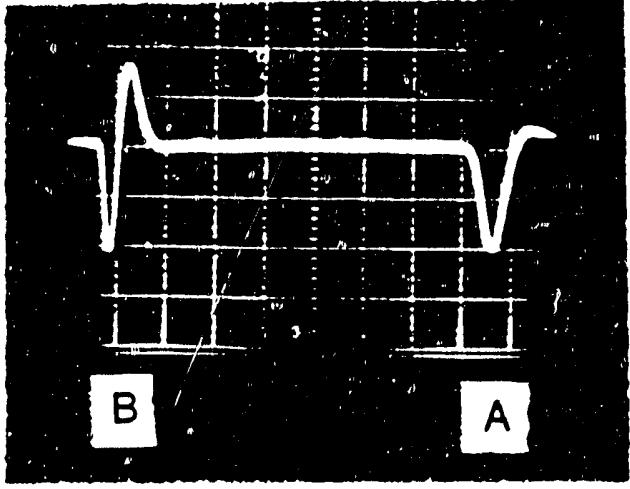

Figure 4: A-pulse corresponding to the collection of the injected charge after an electron drift time of $3.8 \mu \mathrm{s}$. B-injection start pulse.

bulk generation and electron escape through the saddle point or sideways in the z-direction. This dynamic equilibrium is taken into account by the program [5] to calculate the potential within the detector shown in Fig. 3. However, the simulation is only two-dimensional no flow of electrons in the z-direction is possible.

An additional electron falling into the surface region would deform the potential energy upward and one electron would escape into the main valley of the detector or will move sideways. Accumulated electrons are candidates for being injected into the main valley of the detector. Let us assume some negative charge is brought on the metallic gate covering the $\mathrm{SiO}_{2}$ (by applying a negative voltage pulse). This charge closes a part of lines of forces starting on positive charges on the $\mathrm{Si}-\mathrm{SiO}_{2}$ interface. The dynamic equilibrium is disturbed as by a direct injection of electrons across the oxide. The potential in the surface region changes and the equilibrium is restored by injection of excess electrons into the main valley or sideways along the z-direction. After the injection and the return of the gate potential to the original value, the electrons are replenished from the path along the $z$-direction.

We have implemented and tested injectors at a distance of about $2 \mathrm{~cm}$ from the anode. The size of the metal gate was $45 \times 125 \mu \mathrm{m}^{2}$. The drift field was $492 \mathrm{~V} / \mathrm{cm}$ corresponding to an electron drift time of $3.8 \mu \mathrm{s}$. An input rectangular voltage pulse of $700 \mathrm{mV}$ amplitude and $40 \mathrm{~ns}$ width was fed through a high voltage rated $4 \mathrm{nF}$ capacitor to the injector. The DC value of the metal gate was left at its "natural" potential. Fig. 4 shows the waveform of the pulse-shaped anode signal. Pulse A corresponds to about $\mathbf{2 5 0 0 0}$ electrons, equivalent to the charge liberated by the passage of a minimum ionizing particle through the detector. Pulse $B$ is due to the prompt pulser signal applied to the $p^{+}$electrode surrounding the collecting anode. The capacitive coupling of this $p^{+}$electrode to the anode, connected to the low impedance amplifier, accounts for the bipolar shape of $B$. 


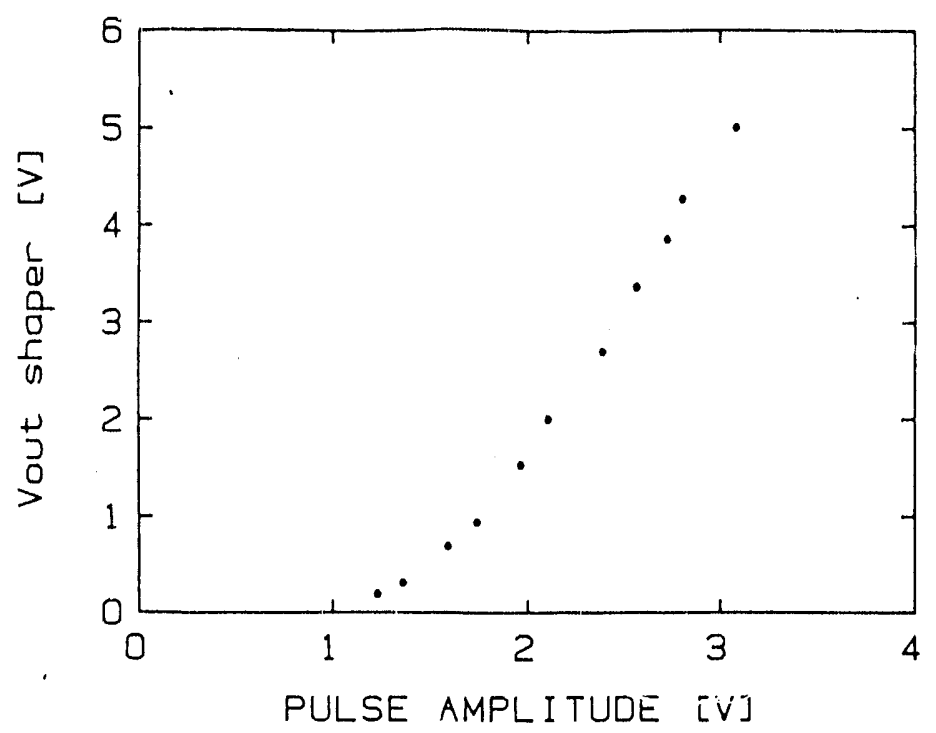

Figure 5: Injected charge as a function of the amplitude of the voltage pulse applied to the injector electrode. The electrode was biased $22 \mathrm{~V}$ below the average value of the adjacent $p^{+}$strips. One minimum ionizing particle corresponds to $0.25 \mathrm{~V}$ at the output.

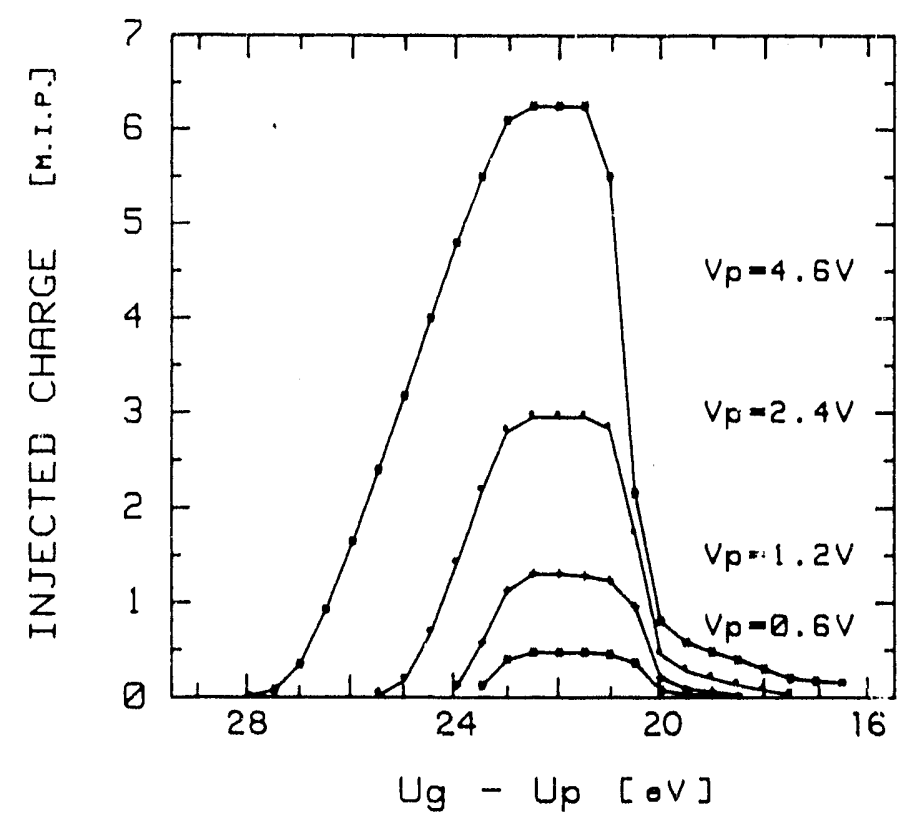

Figure 8: Injected charge in units of 25000 electrons versus injector bias for different pulse amplitudes. Pulse width was constant and equal to $40 \mathrm{~ns}$.

With the DC voltage on the gate of the injector left at its "natural" potential it was possible to control the amount of injected charge by controlling the amplitude of the pulser. The range of injecting charge was between 0.3 and 6 equivalents of minimum ionizing particles.

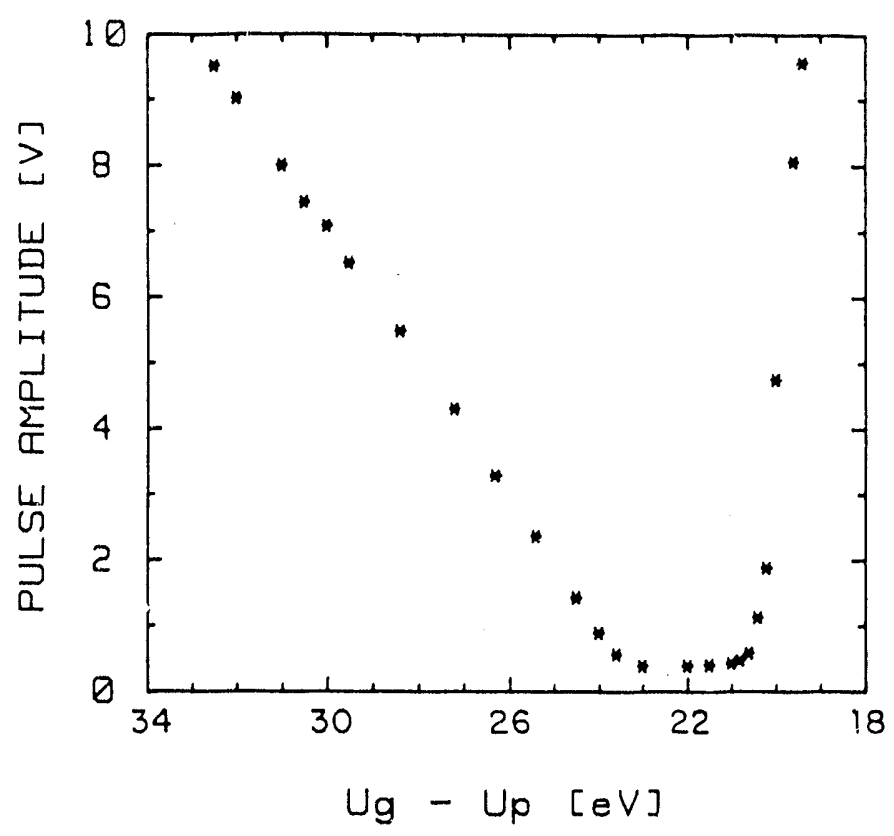

Figure 7: Pulse amplitude at the the injector electrode versus injector bias for fixed injected charge of 10000 electrons.

In order to investigate the behavior of the injector, an external bias was provided to the gate through a $80 \mathrm{k} \Omega$ resistor. At the beginning of the studies such bias was applied at the gate to obtain the same amount of charge as under the "natural" condition. The value of this bias was the "natural" potential of the gate. Its value was about $22 \mathrm{~V}$ below the average negative potential of the neighboring $p^{+}$strips, not far from the value shown in Fig. 3.

Fig. 5 shows the injected charge as a function of the pulse voltage applied to the injector. This injected charge is independent of the bias voltage of the injector within a range of about $2 V$ around the "natural" value as shown in Fig. 6, where the injected charge is plotted versus the injector bias. The amplitude of the pulse is the parameter. Fig. 7 shows the pulse amplitude to be given at the injector electrode to obtain a constant charge of 10000 electrons as a function of the DC bias of the injector electrode. From Fig. 7 we can see that the input pulse does not inject the charge until the threshold voltage is reached. The value of the threshold voltage is close to the "natural" bias of the injector electrode. The observed behaviour is very difficult to explain because it implies a transient analysis of a three-dimensional phenomena. In reality electrons can flow along the zdirection which is not considered in a two-dimensional simulation and not shown in Fig. 3. 


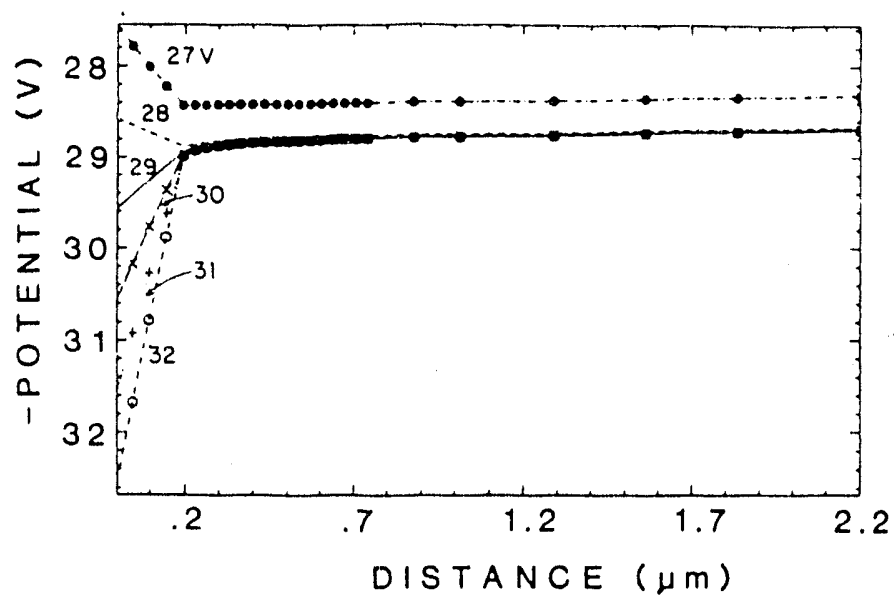

Figure 8: Negative potential in the oxide and in the silicon along the axis of the injector for different biases of the injector electrode. Metal-oxide boundary of the injection electrode is at distance 0 . Oxide extends up to the distance of $0.2 \mu m$ along the $x$-axis. Only first $2 \mu m$ of silicon are shown.

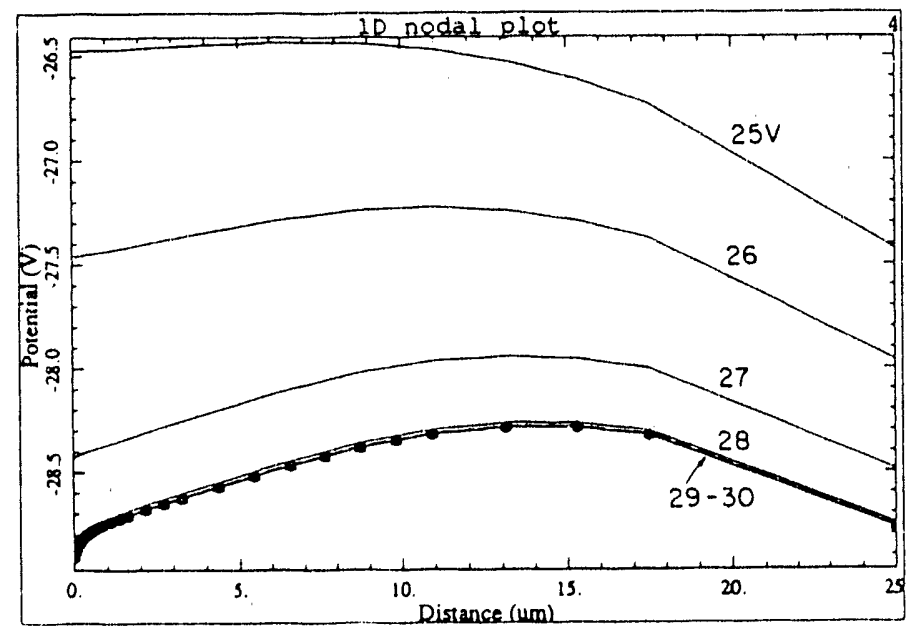

Fingure 9: Negative potential in the silicon on a larger part of the silicon to show the presence of maxima (or saddle points of the potential surface). 'The zero of the $x$-axis is at the $\mathrm{Si}-\mathrm{SiO}_{2}$ interface and the potential wilhin the oxide is not shown.

The pulse repetition rate has been tested up to $0.5 \mathrm{Mhz}$ in the detector, without noticing any rate dependent effects.

\section{B. Steady State Simulation}

We studied with a two-dimensional core [5] the steady state of the injector. Due to the limitation of the two-dimensional code the injector was infinite in the $z$-direction.

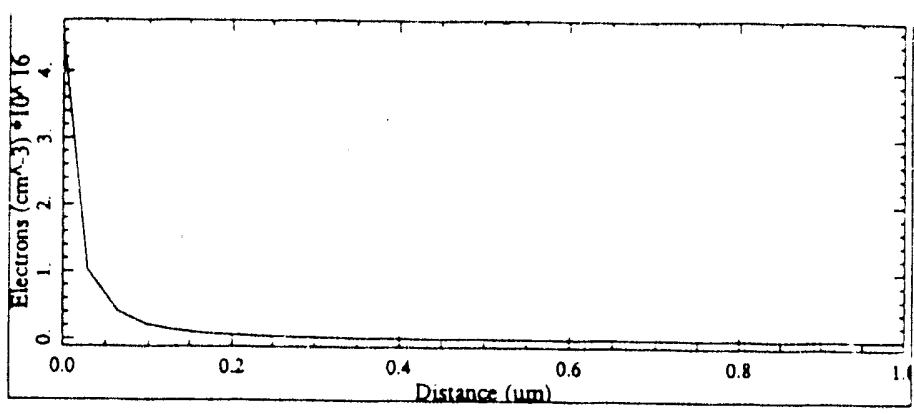

(o)

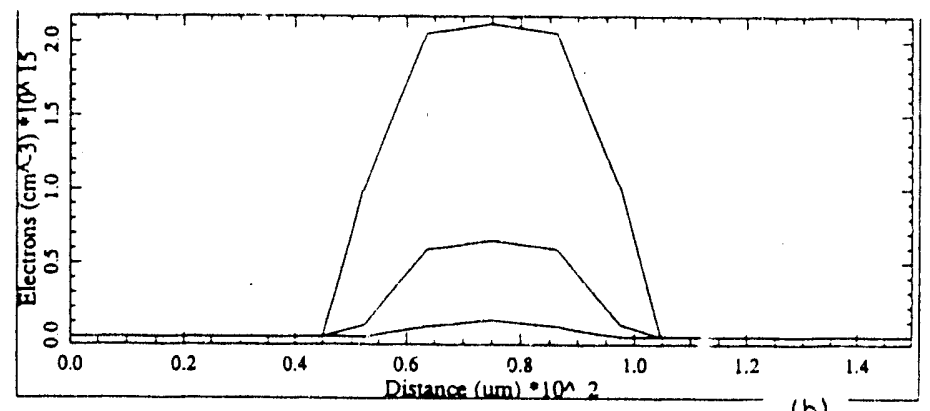

(b)

Figure 10: (a) Distribution of accumulated electrons in silicon near the $\mathrm{Si}-\mathrm{SiO}_{2}$ interface along the injector axis for the injector bias of $29 \mathrm{~V}$. (b) Profiles of accumulated electrons at three different depth below the $\mathrm{Si}-\mathrm{SiO}_{2}$ interface for the same injector bias. The $x$-axis is perpendicular to the $p^{+}$strips. The upper curve is at depth of $0.1 \mu \mathrm{m}$, the middle curve at $0.2 \mu \mathrm{m}$ and the lower curve at $0.5 \mu m$.

Fig. 8 and Fig. 9 show the negative potential in the injector region along the axis of the structure for different biases of the injector electrode. We can see the semiconductors bands at the interface "pinned" at a fixed negative potential of $29 \mathrm{~V}$ below the negative potential of the neighbor $p^{+}$strips for the values of the injector bias below $28 \mathrm{~V}$. The negative potential in silicon also does not change for these values of the injector gate potential. The $n$-type silicon is in accumulation at the interface under the injector electrode and the negative charge of accumulated electrons balances the positive clarge applied to the gate. Under the "pinned" conditions the most of the potential drop is across the oxide.

For the negative bias on the injector electrode higher than $28 \mathrm{~V}$ the bands begin to move at the interface and in the bulk begin to move upwards. For biases higher than about $25 \mathrm{~V}$ the maximum of the negative potential in silicon disappears. Under this condition the electron from the $\mathrm{Si}-\mathrm{SiO}_{2}$ interface can be injected into the bulk of silicon drift detector.

More results of the simulation are shown in Fig. 10 and Fig. 11. 


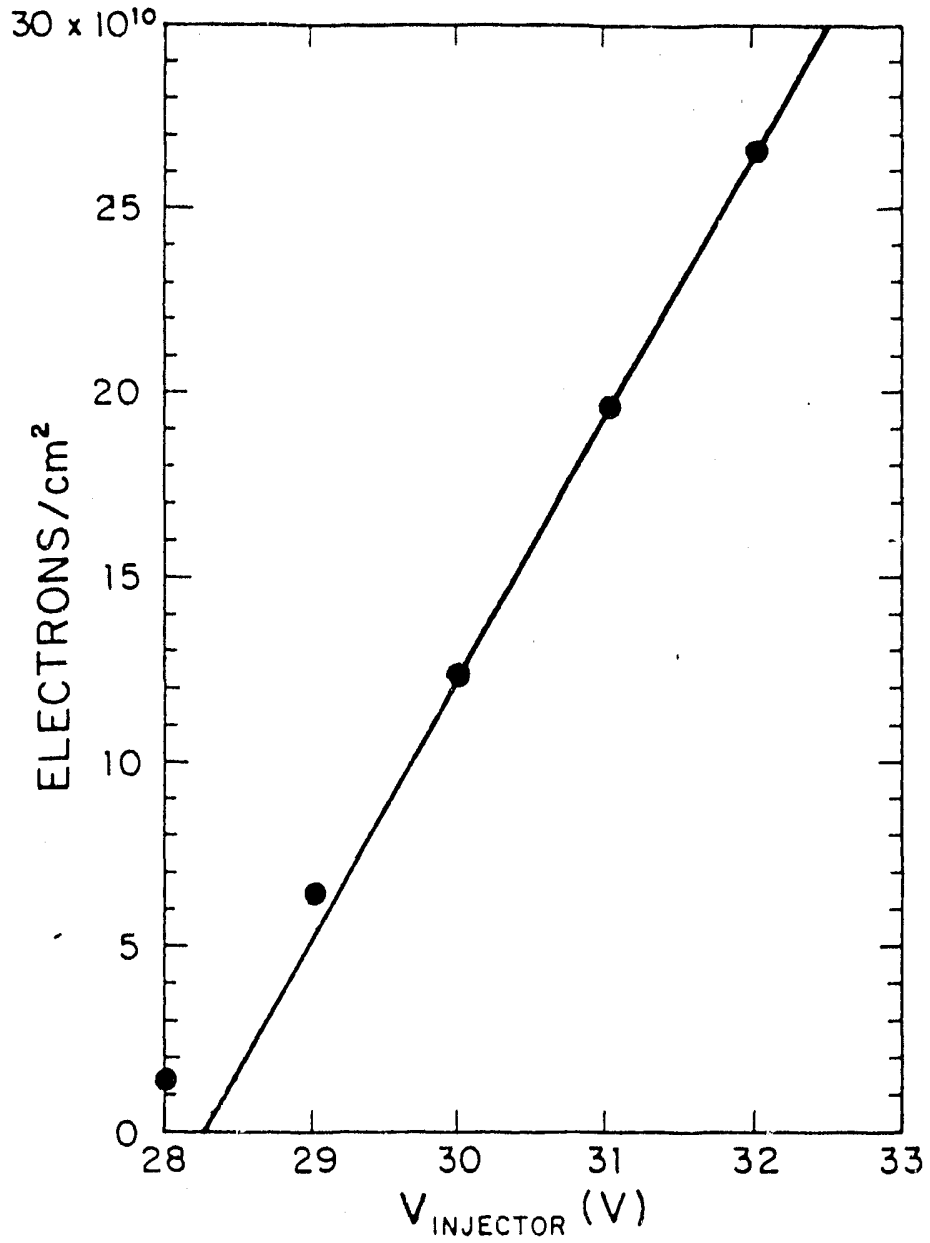

Figure 11: Surface density of electrons accumulated under the electrode of the injector versus the electrode bias. The straight line has the slope corresponding to the capacitance across $0.2 \mu \mathrm{m}$ of the oxide layer.

\section{CONCLUSIONS}

A practical simple device for injecting electrons into silicon drift detectors has been produced and successfully tested. More experimental work and three-dimensional transient simulation code are necessary to fully explain the details of the observed injection mechanism.

\section{Acknowledgments}

The authors wish to thank J. Kemmer and P. Holl for the technological implementation of the silicon drift detector, S. Chinnici and F. Palma for their help during the measurements. E. Gatti and A. Longoni thank V. Radeka for hospitality in BNL.

This manuscript has been althored under contract number DE-AC02-76CH00016 with the U.S. Department of Energy. Accordingly, the U. S. Government retains a non-exclusive, royalty-free licence to publish or reproduce the published form of this contribution, or allow others to do so, for U.S. GOvernment purposes.

$\left(^{*}\right)$ This research is also supported by the Italian INFN, MPI and CNR.

\section{References}

1. E. Gatti and P. Rehak, Nucl. Instr. and Meth. 225, 608 (1984).

2. C. Jacoboni at al., Solid State Electronics, 20,77, (1977).

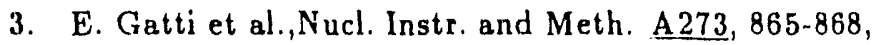
(1988).

4. A. Longoni et al., Nucl. Instr. and Meth. A288, 35-43, (1990).

5. G. Baccarani at al., "HFIELD", Nasecode IV, Dublin, June19-21, 1985.

\section{DISCLAIMER}

This report was prepared as an account of work sponsored by an agericy of the United States Government. Neither the United States Government nor any agency thereof, nor any of their employees, makes any warranty, express or implied, or assumes any legal liability or responsibility for the accuracy, completeness, or usefulness of any information, apparatus, product, or process disclosed, or represents that its use would not infringe privately owned rights. Reference herein to any specific commercial product, process, or service by trade name, trademark, manufacturer, or otherwise does not necessarily constitute or imply its endorsement, recommendation, or favoring by the United States Government or any agency thereof. The views and opinions of authors expressed herein do not necessarily state or reflect those of the United States Government or any agency thereof. 

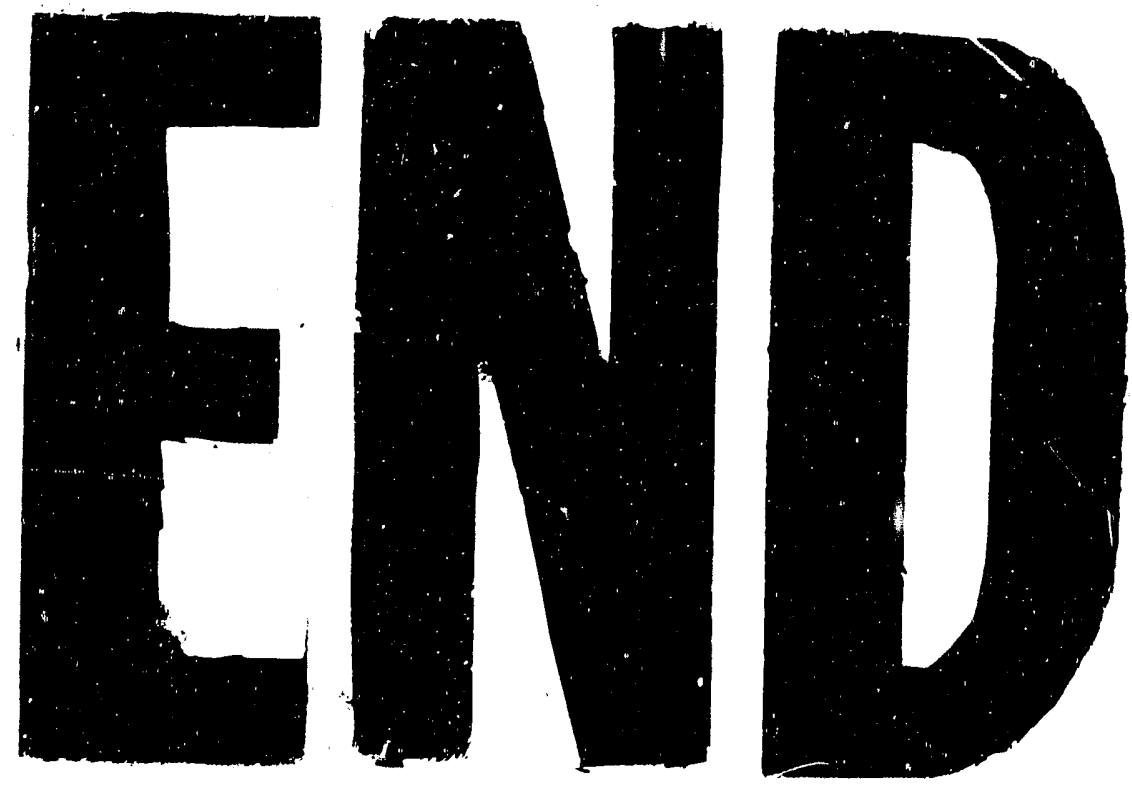

s.
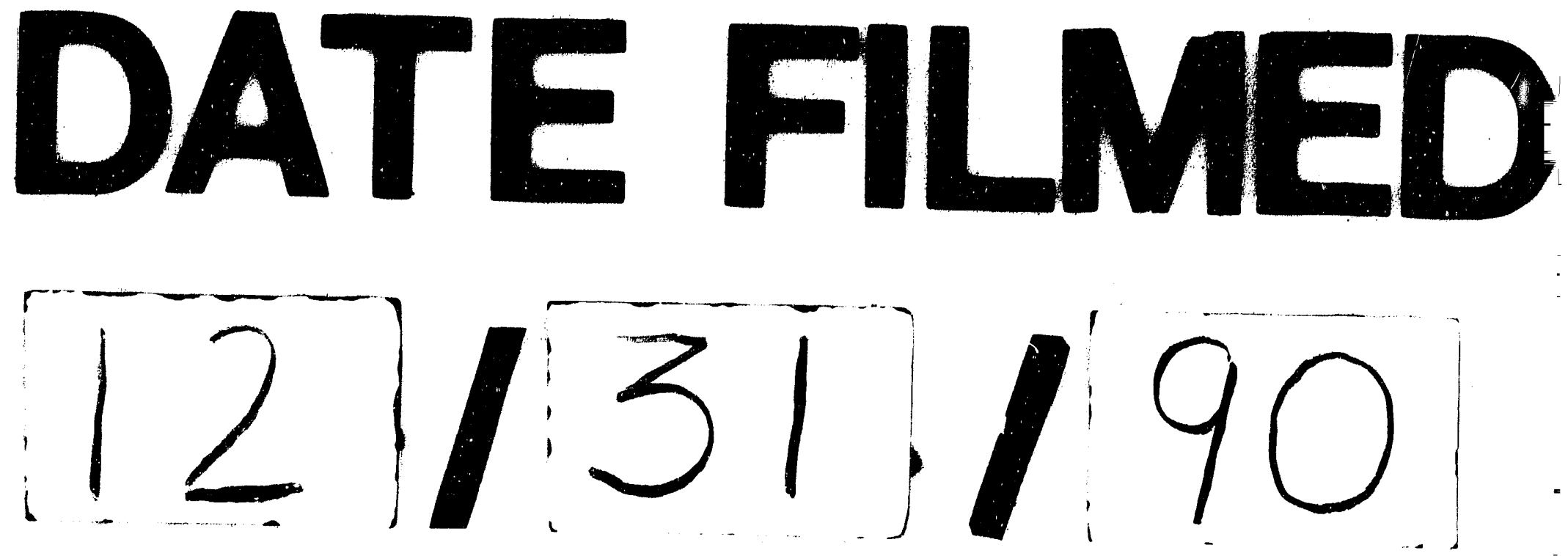
